\title{
APRECIACIÓN CRÍTICA A LA GESTIÓN 2016 Y PERSPECTIVAS AL NUEVO AÑO 2017 DE LA REVISTA PERUANA DE MEDICINA EXPERIMENTAL Y SALUD PÚBLICA
}

\author{
CRITICAL APPRAISAL OF THE 2016 MANAGEMENT AND OVERVIEW OFTHE NEW YEAR 2017 \\ BYTHE REVISTA PERUANA DE MEDICINA EXPERIMENTAL Y SALUD PÚBLICA
}

\author{
Zuño Burstein ${ }^{1, a}$
}

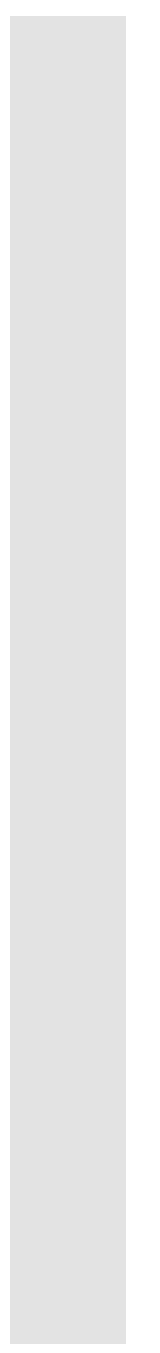

\begin{abstract}
En el informe de gestión 2016 de la Revista Peruana de Medicina Experimental y Salud Pública (RPMESP) que se presentó en enero del presente año el Informe de Gestión 2016 (oficio $N^{\circ}$ 004-2017-CRPMESP/INS) al jefe del Instituto Nacional de Salud (INS) con copia a la ministra de Salud del Perú, se dice que:

Durante el año 2016 se han producido importantes cambios institucionales que han comprometido el funcionamiento, las responsabilidades de los integrantes y la composición del Comité de la Revista, tal como la aceptación de la renuncia del Dr. Ernesto Gozzer Infante a la jefatura del Instituto Nacional de Salud del Ministerio de Salud con fecha 26 de agosto 2016 y nombramiento por Resolución Suprema $N^{\circ}$ 019-2016-SA del nuevo jefe institucional Dr. Luis Antonio Nicolás Suarez Ognio, quien es médico epidemiólogo salubrista, exjefe de la Dirección General de Epidemiologia, con vasta experiencia docente universitaria en varias facultades de medicina peruanas.
\end{abstract}

Así mismo en la composición del gabinete ministerial del nuevo gobierno, la Dra. Patricia J. García Funegra asumió en junio del 2016 el importante cargo de Ministra de Salud. La Dra. García es médico internista, especialista en enfermedades infecciosas y exdecana de la Facultad de Salud Pública y Administración de la Universidad Peruana Cayetano Heredia; además, es miembro de nuestro Consejo Consultivo, exjefa del INS y Académica de la Academia Nacional de Medicina (ANM), condiciones que permiten tener la confianza del apoyo que el gobierno central otorgará a nuestra labor.

El distinguido economista Pedro Pablo Kuczynski Godard fue elegido, democráticamente, como presidente de la República de Perú y asumió el cargo en julio del año pasado. Es hijo de una de las personalidades más destacadas de la salud pública en el Perú, el Dr. Maxime Hans KuczynskiGodard, quien fue médico patólogo salubrista, nacido en Alemania en el año de 1890, con quien el actual presidente peruano ha manifestado reiteradamente su profunda identificación con su pensamiento y con su invalorable contribución en beneficio de nuestro país.

El Dr. Máxime Kuczynski-Godard, luego de desempeñar una destacada labor de investigación y educación en la Universidad de Berlín a través de numerosas misiones médicas en diferentes

\footnotetext{
1 Instituto Nacional de Salud. Lima, Perú.

a Director de la RPMESP

Recibido: 17/02/2017 Aprobado: 22/02/2017 En línea: 23/03/2017
}

Citar como: Burstein Z. Apreciación crítica a la gestión 2016 y perspectivas al nuevo año 2017 de la Revista Peruana de Medicina Experimental y Salud Pública. Rev Peru Med Exp Salud Publica. 2017;34(1):7-10. doi: 10.17843/rpmesp.2017.341.2761 
partes del mundo, tuvo que abandonar Alemania a la llegada del nazismo al poder. Fue acogido en el Perú el año 1936 por el Dr. Carlos Enrique Paz Soldán en el Instituto de Medicina Social de la Universidad Nacional Mayor de San Marcos (UNMSM) y posteriormente por el Dr. Constantino Carvallo, Ministro de Salud, quien le encargó importantes responsabilidades sanitarias en la Amazonía peruana en donde realizó y publicó numerosos trabajos científicos en colaboración, entre otros, con el Prof. Dr. Hugo Pesce en el control de la lepra. Es destacable en sus investigaciones su autoinoculación experimental de la verruga peruana realizada con un gran rigor científico, controlada por el Dr. Daniel Mac Kehni, reproduciendo la experiencia de Daniel A. Carrión; ese trabajo le valió la designación de Miembro Honorario de nuestra ANM ${ }^{(1)}$.

Su profunda y permanente vocación de servicio social le permitió expresar que "el médico para ser lo que debe ser, no puede limitarse a ser curaciones o esperar que se le presenten enfermos; ha de penetrar en el medio, ha de interesarse en todos los aspectos de la vida del pueblo cuya protección es su propósito"(2). Es de esperar que el actual presidente del Perú haya heredado estos principios de su padre.

El Dr. Máxime Kuczynski-Godard recibió numerosos homenajes recordatorios de intelectuales y personalidades científicas como las otorgadas en su momento por el Dr. José Neyra Ramírez en la Sociedad Peruana de Enfermedades Tropicales e Infecciosas, del Dr. Fausto Garmendia Lorena en la Sociedad Medica Peruana Alemana, del Dr. Marcos Cueto en diferentes publicaciones; y de nosotros en la RPMESP en una sección especial en el año 2003, destacando su labor como pionero en la Salud Publica (1) y a través de una galería fotográfica muy bien lograda en el año 2010 con la autoría de los Dres. Michael Knipper de la Universidad Giessen de Alemania y Oswaldo Salaverry de la UNMSM (3). En el año 2013, el INS y la UNMSM crearon en la ciudad amazónica de lquitos el Centro de Investigaciones de Enfermedades Tropicales, que lleva el nombre de "Hugo Pesce - Máxime Kuczynski", en homenaje y reconocimiento a esas dos personalidades científicas que contribuyeron decididamente al estudio y control de las enfermedades tropicales en el Perú.

Durante el año 2016 se han producido algunos cambios importantes en el equipo de trabajo del Comité Editor de la RPMESP, entre ellos está la reincorporación del médico internista Dr. Alonso Soto Tarazona, de amplia experiencia en publicaciones médicas, como editor científico para el periodo 2016-2018 según Resolución Jefatural del 13 de junio del 2016; asimismo, el médico Hugo Arroyo Hernández quién que se desempeñaba como editor científico hasta el 13 de junio del 2016 se mantiene como editor adjunto hasta el 2018, junto con el médico Rubén Borja García también como editor adjunto desde el mes de febrero del 2016 y de manera oficial a partir del 13 de junio del 2016. Completando el grupo de editores adjuntos, el médico Juan Jhonnel Alarco Urquizo fue ratificado como editor adjunto con responsabilidad de coordinador de la Oficina Funcional de Publicaciones; el médico Jimmy Carreazo Pariasca asumió la coordinación administrativa y es responsable de la distribución de la Revista. La Lic. Bertha Huarez Sosa se mantiene como asistente editorial y con la importante función de responsable técnico-secretarial con el apoyo de una secretaria asignada al comité, finalmente, el Lic. Daniel Cárdenas Rojas se mantiene como corrector de estilo.

Este importante grupo de trabajo técnico-administrativo profesional es el directamente responsable, bajo la orientación del director Dr. Zuño Burstein Alva y del editor general Dr. César Cabezas Sánchez representante de la Jefatura del INS, de todo el proceso editorial, con la colaboración de los miembros del Comité Editor de la RPMESP y de los miembros del Consejo Consultivo, a quienes se les solicita y agradece la generosa y valiosa colaboración ad honoren para mantener la alta calidad lograda a través de años de esfuerzo de la RPMESP ubicándola como la mejor revista médico-científica peruana, y una de las mejores latinoamericanas.

Se reitera lo expresado, ahora actualizado y agravado, en el Editorial del primer número de la RPMESP del año pasado que ${ }^{(4)}$ : Es seria y preocupante la situación de crisis en el Perú y en el mundo, existe inestabilidad institucional, inseguridad, corrupción y quebrantamiento de valores fundamentales... Todo lo expresado, agregado a las amenazas de catástrofes naturales condicionadas por el cambio climático que se predice, afectará seriamente a nuestro país con repercusión en la salud pública en los próximos meses del presente año; por otra parte, la peligrosa aparición de epidemias de enfermedades transmitidas por mosquitos vectores, como el dengue, el chikungunya, zika y otras afecciones emergentes y reemergentes, obligan a las instituciones como la Academia Nacional de Medicina y el Instituto Nacional de Salud, a intensificar su participación, 
cumpliendo la función que le corresponde al INS como ejecutor tecnológico, y de reserva moral y académica a la ANM, asesorando, además a los responsables políticos sobre problemas de su competencia en beneficio de la comunidad.

El actual jefe del INS, Dr. Luis Suarez, se ha comprometido formalmente a seguir apoyando a la RPMESP; sin embargo, por razones presupuestales, la versión electrónica a texto completo en idioma ingles ha quedado postergada, para oportunidad posterior que se espera sea a corto plazo en beneficio de la visibilidad que debe tener nuestra publicación. Hemos insistido, además, en que la estructura administrativa de la RPMESP se constituya como una unidad operativa orgánica, con un nivel remunerativo justo para sus integrantes que incentive a sus ejecutores y dé estabilidad a esta indispensable publicación de difusión científica del INS, brazo científico del Ministerio de Salud peruano puesto al servicio de la investigación médica científica y de la salud del Perú y Latinoamérica.

La RPMESP ha cumplido satisfactoriamente con su cronograma de publicación del año 2016; durante dicho año se han recibido 448 artículos, de los cuales fueron aprobados 74 y 305 no merecieron la aprobación para su publicación, los restantes aún se encuentran el proceso de edición. De los artículos presentados, 238 correspondieron a trabajos de investigación científica; 351 correspondieron al Perú, 265 de ellos de Lima, uno del Callao y 85 de Provincias. El total de artículos presentados de autores extranjeros fue 97, de los siguientes países: Alemania, Argentina, Brasil, Canadá, Chile, Colombia, Costa Rica, Cuba, EE.UU., España, Guatemala, Honduras, Italia, México, Paraguay, Reino Unido, Uruguay y Venezuela. Siendo los más numerosos de Colombia, Argentina, México, Cuba, Ecuador, España, Chile, Brasil, Venezuela y EE. UU.

Durante el año 2016 se programó y público en cada número de la RPMESP, los siguientes temas que dieron origen a sendas conferencias conjuntas con la ANM: 1. Impacto del Cambio Climático sobre la Salud con el editor invitado Dr. Rubén Figueroa, médico perteneciente al grupo de asesores del MINSA, realizada el 7 de abril de 2016, con 91 asistentes. 2. Salud y Calidad de Vida en el Adulto Mayor con el editor invitado Dr. Luis Varela, médico geriatra, profesor de la UPCH y secretario científico de la ANM, realizada el 7 de julio de 2016 con 170 asistentes. 3. Hacia un Cambio del Sistema de Salud Peruano, Experiencias y Perspectivas con el editor invitado Dr. Aníbal Velásquez, médico salubrista, profesor de la UNMSM, exministro de Salud y miembro del Consejo Consultivo de la RPMESP, realizada el 29 de setiembre de 2016 con 104 asistentes. 4. Medicina Centrada en la persona del concepto a la acción con el editor invitado Dr. Alberto Perales, médico psiquiatra, profesor de la UNMSM, expresidente de la ANM y miembro del Comité Editor de la RPMESP; realizada el 7 de diciembre de 2016 con 59 asistentes.

El grupo de trabajo del Comité de la RPMESP organizó y dicto el Curso Precongreso: "Investigación y redacción científica biomédica" en el marco del Congreso Científico Internacional del INS realizado el 15 de noviembre de 2016 con una asistencia de 231 personas.

Para el año 2017 se han programado y comprometido los siguientes temas para los cuatro números de la RPMESP: "Obesidad como problema de salud pública" con el editor invitado Dr. Miguel Malo de la Organización Panamericana de la Salud, la recepción de artículos para este número se cerró el 4 de enero de 2017 y la versión impresa de la Revista está saliendo oportunamente al término del primer trimestre del año en curso, en tanto la Conferencia Conjunta con la ANM y la presentación pública de la Revista se ha programado para el 25 de abril de 2017. El segundo número tiene como tema principal: "Antropología de la Salud" con el editor invitado Dr. Michael Knipper de la Universidad Giessen de Alemania, la recepción de artículos para ese número vence el 15 de marzo y la Conferencia Conjunta con la ANM y presentación pública de la Revista será el 20 de julio del 2017. El tercer número dedicará su tema principal a: "Evaluación de Programas de Salud" con el editor invitado Dr. Alfonso Gutiérrez, del Ministerio de Economía y Finanzas como editor invitado, la fecha de cierre de recepción de artículos es el 7 de junio de 2017 y la presentación el 14 de septiembre de 2017. Por último el cuarto tema será "Anemia Infantil" con la editora invitada Dra. Nelly Zavaleta, directora general del Centro Nacional de Alimentación y Nutrición del INS; la recepción de artículos será hasta el 6 de setiembre del 2017 y tanto la presentación pública como la Conferencia Conjunta se realizarán el 7 de diciembre de 2017.

La Revista Peruana de Medicina Experimental y Salud Pública del INS reitera que se encuentra abierta para la publicación de artículos que contribuyan a la salud pública, sean estos para cualquiera de sus diferentes 
secciones, además de los comprometidos y enviados para el tema principal de cada número; los trabajos enviados deberán cumplir con los requerimientos exigidos en las Instrucciones para la Presentación de Artículos a la Revista Peruana de Medicina Experimental y Salud
Publica, adjuntar los formatos correspondientes y serán aprobados para su publicación después de pasar por el proceso editorial correspondiente, con la calificación por pares de expertos nacionales e internacionales, a quienes agradecemos por su valiosa colaboración.

\section{REFERENCIAS BIBLIOGRÁFICAS}

1. Kuczynski-Godard M. La autoexperiencia del prof. Max. Kuczynski-Godard. La Reforma Médica. 1937;23(267):758-78.

2. Burstein Z. Máxime KuczynskiGodard, un pionero de la Salud Pública.
Rev Peru Med. Exp Salud Publica. 2003;20(4):231.

3. Knipper M. Salaverry O. Máxime Kuczynski-Godard (1980-1967). Fotografías médicoantropológicas de la amazonia y de los andes peruanos, 1938-
1948. Rev Peru Med. Exp Salud Publica. 2010;27(1):146-50.

4. Burstein Z, Cabezas C. La Revista Peruana de Medicina Experimental y Salud Pública continúa al servicio de la Salud Pública del Perú y Latinoamérica. Rev Peru Med. Exp Salud Publica. 2016;33(1):10-2.

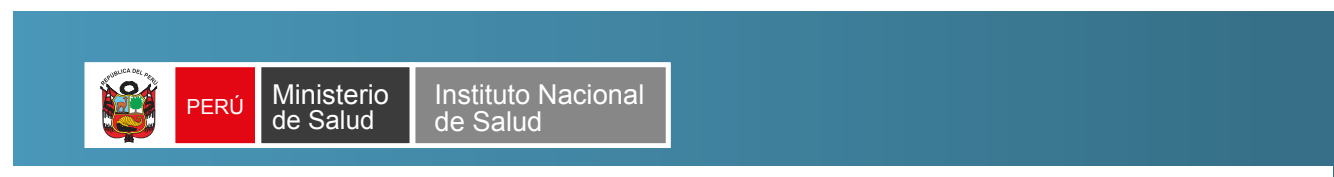

\section{Inclusión social en salud: acercando el diagnóstico de dengue a las poblaciones afectadas}

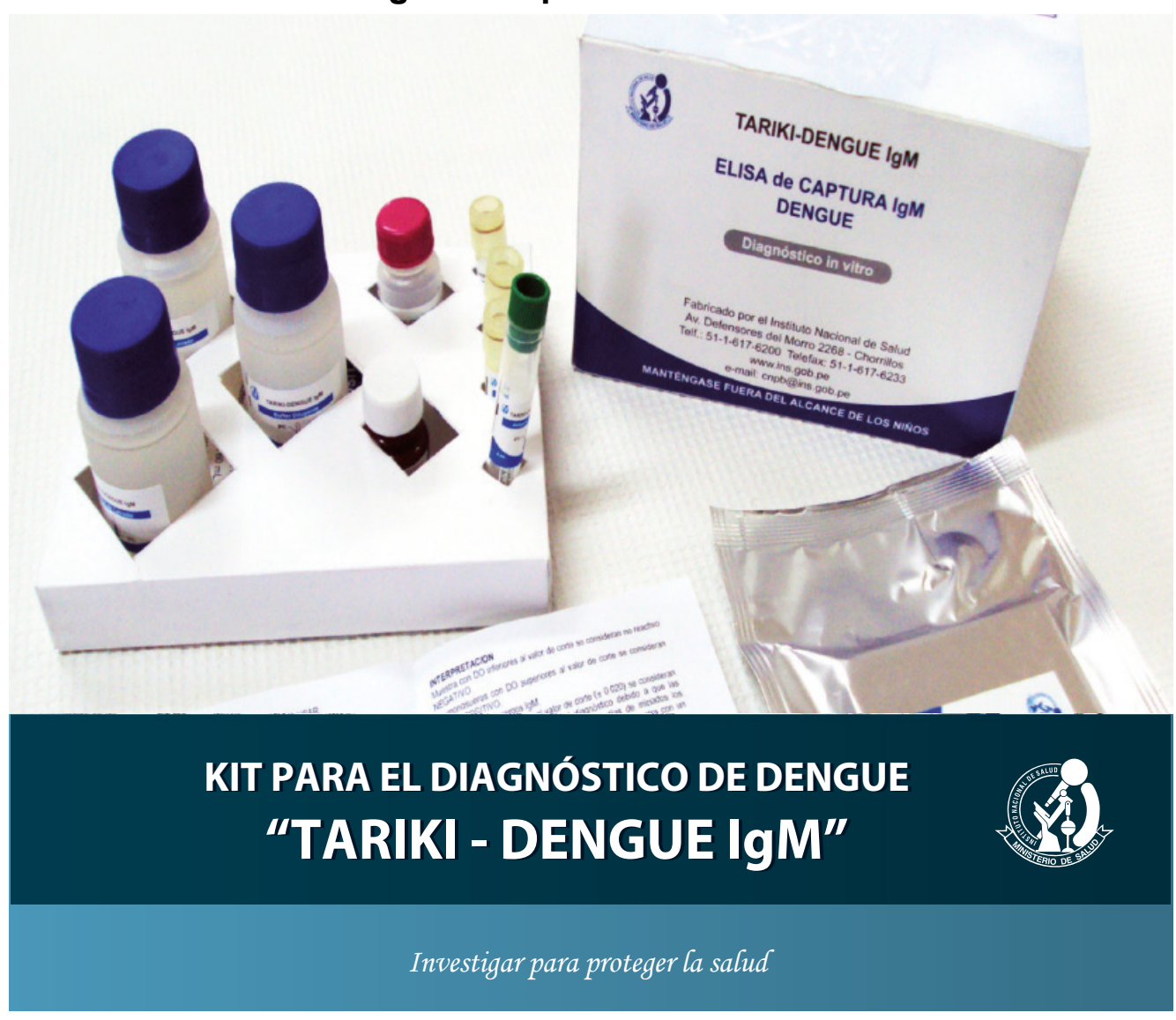

